\title{
Lifetime Cocaine and Opiate Use and Chronic Kidney Disease
}

\author{
Tessa Novick $^{a}$ Yang Liu $^{a}$ Anika Alvanzo $^{a}$ Alan B. Zonderman ${ }^{b}$ \\ Michele K. Evans ${ }^{\mathrm{b}}$ Deidra C. Crews ${ }^{\mathrm{a}}$ b \\ ${ }^{a}$ Department of Medicine, Johns Hopkins University School of Medicine, ${ }^{b}$ National Institute on Aging, National \\ Institutes of Health, and 'Welch Center for Prevention, Epidemiology and Clinical Research, Johns Hopkins Medical \\ Institutions, Baltimore, Md., USA
}

\section{Key Words}

Chronic kidney disease $\cdot$ Substance abuse · Epidemiology · Cocaine $\cdot$ Opiates

\begin{abstract}
Background: More than $50 \%$ of American adolescents and adults report having used illicit drugs in their lifetime. We examined the association of lifetime opiate and cocaine use with reduced kidney function, albuminuria and rapid kidney function decline among urban-dwelling adults. Methods: Our prospective cohort included 2,286 Healthy Aging in Neighborhoods of Diversity across the Life Span study participants who were community-dwelling adults residing in Baltimore, MD. The predictive variables were lifetime opiate and cocaine use, defined as use of opiates or crack/cocaine $\geq 5$ times. Outcomes included prevalent reduced estimated glomerular filtration rate (eGFR; $<60 \mathrm{ml} / \mathrm{min} / 1.73 \mathrm{~m}^{2}$ by Chronic Kidney Disease (CKD)-Epidemiology Collaboration), albuminuria (albumin-to-creatinine ratio $>30 \mathrm{mg} / \mathrm{g}$, $\mathrm{n}=1,652)$ and rapid kidney function decline $(>3 \mathrm{ml} /$ $\mathrm{min} / 1.73 \mathrm{~m}^{2}$ per year over a median of 4.7 years, $\mathrm{n}=1,660$ ). Results: Participants' mean age was 48 years, $15 \%$ reported opiate use, and $22 \%$ reported cocaine use. A total of 115 (5.0\%) participants had reduced eGFR, 190 (11.5\%) had albuminuria and 230 (13.8\%) experienced rapid decline in kidney function. In adjusted logistic regression analyses, both substances were associated with greater odds of reduced eGFR
\end{abstract}

(OR 2.71, 95\% Cl 1.50-4.89 for opiates; OR 1.40, 95\% Cl 0.872.24 for cocaine). Both substances were associated with greater odds of albuminuria (OR $1.20,95 \% \mathrm{Cl} 0.83-1.73$ for opiates; OR 1.80, 95\% Cl 1.29-2.51 for cocaine). Neither substance was associated with the rapid decline of kidney function. Conclusions: Lifetime opiate and cocaine use was associated with prevalent reduced eGFR and albuminuria, yet not with rapid kidney function decline. The use of opiate and cocaine may be an important risk factor for CKD in urban populations.

(c) 2016 S. Karger AG, Basel

\section{Introduction}

In 2014, more than 130 million Americans over age 12 (49\%) reported the use of illicit drugs during their lifetime, including cocaine, heroin, hallucinogens, inhalants, marijuana, and prescription pain-killers [1]. However, little is known about the relationship between lifetime illicit drug use and kidney functions.

According to the most recent National Survey on Drug Use and Health, 14.8 and 3.6\% of Americans endorsed lifetime use of cocaine and opioids, respectively [1]. Among those who endorsed lifetime use of opioids, $13.6 \%$ reported non-medical use of pain relievers and $1.8 \%$ reported use of heroin [1]. The pattern of illicit drug use varies widely based on the geographic location, and in

\section{KARGER}

E-Mail karger@karger.com

www.karger.com/ajn
(C) 2016 S. Karger AG, Basel

0250-8095/16/0446-0447\$39.50/0
Tessa Novick, MD

Department of Medicine, Johns Hopkins University School of Medicine 4940 Eastern Avenue, 301 Building

Suite 3400, Baltimore, MD 21224 (USA)

E-Mail tnovick1@jhmi.edu 
Baltimore, Maryland, heroin use represents a significant public health concern [2-4]. The Baltimore Mayor's Heroin Task Force estimated that nearly 19,000 individuals in Baltimore used heroin in 2014 [4]. The impact of this problem is reflected in Baltimore's heroin associated overdose death rate, which rose by $21 \%$ during the period from 2013 to 2014, and is 8 times the rate of death that occurs due to motor vehicle accidents $[4,5]$. The majority of Baltimore's overdose-related deaths in 2014 were related to the use of heroin, and approximately one third were related to cocaine use [3].

Baltimore is among the top 25 cities in the United States with regard to the incidence and prevalence of endstage renal disease (ESRD) [6]. While many studies have identified putative risk factors for kidney disease, connections between opiate and cocaine use and kidney disease remain uncertain $[7,8]$. Given the impact of opiate and cocaine use on kidney disease in urban areas like Baltimore, it is important to elucidate if use of these substances increases the risk for kidney disease. We sought to determine the association of opiate and cocaine use with prevalent reduced estimated glomerular filtration rate (eGFR), albuminuria and rapid kidney function decline among a prospective cohort of urban-dwelling adults. Understanding whether opiate and cocaine use is a modifiable risk factor for adverse kidney outcomes could further enable the development of more targeted interventions and public policies for this population.

\section{Methods}

\section{Study Design}

We examined data from the National Institute on Aging, Healthy Aging in Neighborhoods of Diversity across the Life Span (HANDLS) study. HANDLS is a population-based cohort study about the influences and interaction of race and socioeconomic status (SES) on the development of cardiovascular and cerebrovascular health disparities among minority and lower SES subgroups [9]. HANDLS participants include white and black individuals between the ages of 30 and 64 who live in 13 census tracts in Baltimore City, Maryland. Household enrollment was from August 2004 to November 2008. Each participant provided written informed consent. The National Institute of Environmental Health Sciences, National Institute of Health approved the study protocol.

\section{Participants}

This study was limited to HANDLS participants for whom baseline serum creatinine values were known; they responded to questions about opiate and cocaine use. Of 3,720 HANDLS participants, 1,066 were excluded due to absence of data on kidney function, and 368 were excluded due to absence of data on opiate and cocaine use; thus, only 2,286 participated in the baseline analysis. Of 2,286 participants in the baseline analysis, 621 (27.2\%) par- ticipants were excluded due to absence of data on eGFR decline, leaving 1,665 for rapid eGFR decline analysis. Of those 2,286, $634(27.7 \%)$ were excluded due to absence of data on urine albumin-to-creatinine ratio. Compared to those who were excluded, participants included in the baseline analysis $(\mathrm{n}=2,286)$ were older ( 48 vs. 47 years), and more likely to be female (56 vs. $52 \%$ ) and white ( 43 vs. $38 \%$; p value $<0.05$ for all); poverty status was the only common factor between the two groups. Included participants were less likely to report lifetime use of cocaine and opiates than those who were excluded due to the absence of creatinine information ( 22 vs. $29 \%$ for cocaine, and 15 vs. $25 \%$ for opiate use among included and excluded participants, respectively).

\section{Measurements and Definitions}

All participants underwent an initial household survey and then a medical assessment in a mobile research vehicle (MRV). The household survey included self-reported information on demographics and SES. The MRV medical assessment was obtained by healthcare providers, and included a medical history, substance use history, physical exam, and baseline laboratory measurement [9-11]. Independent variables of interest included opiate and cocaine use, which were self-reported at the time of medical assessment. Opiate use was defined as lifetime use of heroin, morphine and/or codeine $\geq 5$ times. Cocaine use was defined as lifetime use of cocaine and/or crack $\geq 5$ times regardless of the route of ingestion. In the sensitivity analysis, current opiate and cocaine use was defined as the use within 1 month of the interview.

Dependent variables included prevalent reduced eGFR, albuminuria, and rapid kidney function decline. eGFR was calculated using the Chronic Kidney Disease (CKD)-Epidemiology Collaboration creatinine-based equation for calibrated serum creatinine [12]. Baseline creatinine was measured once at the baseline medical assessment (HANDLS wave 1). Follow-up creatinine was obtained during the HANDLS wave 3 assessment. Reduced eGFR was defined as baseline eGFR $<60 \mathrm{ml} / \mathrm{min} / 1.73 \mathrm{~m}^{2}$. Serum creatinine was measured for $8 \%$ of participants at the NIA Clinical Research Branch Core Laboratory using modified kinetic Jaffe method (CREA method, Dade Dimension X-P and Clinical Chemistry System, Siemens Healthcare Diagnostics Inc., Newark Del., USA) and was measured for the remaining participants at Quest Diagnostics, Inc., by isotype dilution mass spectrometry (Olympus America Inc., Melville, N.Y., USA) and standardized to the reference laboratory at the Cleveland Clinic. Albuminuria was defined as urine albumin-to-creatinine ratio $>30 \mathrm{mg} / \mathrm{g}$ using baseline urine measurements. Urine albumin concentration was measured at Quest Diagnostics, Inc., using an immunoturbimetric assay (Kamiya Biomedical Co., Seattle, Wash., USA). Rapid kidney function decline was defined as reduction in eGFR $>3 \mathrm{ml} / \mathrm{min} / 1.73 \mathrm{~m}^{2}$ per year over a median of 4.7 years of follow-up [13].

Covariates included demographic and SES information, such as race (black or white), age, gender, health insurance status, poverty status (income above or below $125 \%$ of the federal poverty level), and highest completed grade level. Additional covariates included health behaviors and medical conditions, such as problem drinking, cigarette use, hypertension, diabetes mellitus, HIV, and hepatitis $C$ and $B$ infection. Problem drinking was defined as the use of alcohol in the last 6 months in the setting of (A) being told to stop by a health care professional, or (B) during responsibilities such as work, school or taking care of children. Individuals were classified as smokers if they reported current tobacco use. 
Table 1. Participant characteristics, by opiate and cocaine use* groups $(\mathrm{n}=2,286)$

\begin{tabular}{|c|c|c|c|c|c|c|}
\hline \multirow[t]{3}{*}{ Participant characteristics } & \multicolumn{6}{|l|}{ Drug use* } \\
\hline & \multicolumn{3}{|l|}{ opiate } & \multicolumn{3}{|l|}{ cocaine } \\
\hline & $\begin{array}{l}\text { user } \\
(\mathrm{n}=342 ; 15 \%)\end{array}$ & $\begin{array}{l}\text { non-user } \\
(\mathrm{n}=1,944 ; 85 \%)\end{array}$ & $\mathrm{p}$ value & $\begin{array}{l}\text { user } \\
(\mathrm{n}=501 ; 22 \%)\end{array}$ & $\begin{array}{l}\text { non-user } \\
(\mathrm{n}=1,785 ; 78 \%)\end{array}$ & $\mathrm{p}$ value \\
\hline Age, mean (SD) & $47(7)$ & $48(9)$ & 0.006 & $48(7)$ & $48(10)$ & 0.124 \\
\hline Gender, male & $225(66)$ & $775(40)$ & $<0.001$ & $296(59)$ & $704(39)$ & $<0.001$ \\
\hline African American race & $261(76)$ & $1,051(54)$ & $<0.001$ & $323(65)$ & $989(55)$ & $<0.001$ \\
\hline IV drug use & 170 & & & 113 & & \\
\hline $\mathrm{HBV}$ & $30(9)$ & $41(2)$ & $<0.001$ & $39(8)$ & $32(2)$ & $<0.001$ \\
\hline $\mathrm{HCV}$ & $101(30)$ & $54(3)$ & $<0.001$ & $108(22)$ & $47(3)$ & $<0.001$ \\
\hline HIV & $4(2)$ & $26(2)$ & 0.843 & $8(2)$ & $22(2)$ & 0.548 \\
\hline Hypertension & $147(43)$ & $953(49)$ & 0.038 & $231(46)$ & $869(49)$ & 0.303 \\
\hline Diabetes & $49(14)$ & $343(18)$ & 0.141 & $62(12)$ & $330(19)$ & 0.001 \\
\hline Current smoking & $256(76)$ & $841(44)$ & $<0.001$ & $348(70)$ & $749(42)$ & $<0.001$ \\
\hline Problem drinking & $64(24)$ & $205(14)$ & $<0.001$ & $105(26)$ & $164(12)$ & $<0.001$ \\
\hline Insurance & $193(57)$ & $1,318(70)$ & $<0.001$ & $294(61)$ & $1,217(70)$ & $<0.001$ \\
\hline Poverty & $190(56)$ & $730(38)$ & $<0.001$ & $251(50)$ & $669(38)$ & $<0.001$ \\
\hline Education, years, mean (SD) & $11(2)$ & $13(3)$ & $<0.001$ & $12(3)$ & $13(3)$ & $<0.001$ \\
\hline
\end{tabular}

* Opiate and cocaine use: defined as self-reported lifetime use of opiate (heroin, morphine and/or codeine) or cocaine (cocaine and/ or crack) $\geq 5$ times, regardless of the route of ingestion.

$\mathrm{HBV}=$ Hepatitis B virus; $\mathrm{HCV}=$ hepatitis $\mathrm{C}$ virus; HIV = human immunodeficiency virus.

Participants underwent sitting and standing blood pressure measurements on each arm using the brachial artery auscultation method. Hypertension was defined as systolic blood pressure $\geq 140 \mathrm{~mm} \mathrm{Hg}$ or diastolic blood pressure $\geq 90 \mathrm{~mm} \mathrm{Hg}$, (obtained using an average of seated and standing systolic and diastolic blood pressures), a history of blood pressure medication use, or self-report of hypertension. Diabetes mellitus was defined as fasting plasma glucose concentration of $\geq 126 \mathrm{mg} / \mathrm{dl}$ ( $7.0 \mathrm{mmol} / \mathrm{l})$, self-report of diabetes or history of diabetic medication use. HIV status was measured using an HIV-1/HIV-2 enzyme-linked immunoassay. Hepatitis $\mathrm{B}$ and $\mathrm{C}$ viruses were defined as patients self-reporting of having been affected by this infection in the past.

\section{Statistical Analysis}

Participant characteristics were compared according to the substance use status using chi-square tests or Fisher exact tests for categorical variables and analysis of variance tests for continuous variables. Multivariable logistic regression was performed to determine the presence, direction and magnitude of the association between substance use status and reduced eGFR, albuminuria and rapid kidney function decline. Confounders included in the multivariable models were age, gender, race, poverty status, insurance status, problem drinking, smoking status, and comorbidities (hepatitis B, hepatitis C, HIV status, hypertension and diabetes).

In all analyses, we controlled the possibility of confounding by US census tract using the fixed effects modeling, clustered on neighborhood. Statistical analyses were performed using STATA software, version 13 (StataCorp, College Station, Tex., USA). A two-sided $p<0.05$ was used as the level of significance for all tests.

Opiate and Cocaine Use and CKD in Urban Adults

\section{Sensitivity Analysis}

In a sensitivity analysis, we used current opiate and/or cocaine use as the independent variables of interest, and examined their association with reduced eGFR, albuminuria and rapid kidney function decline. To assess the impact of potential laboratory variability, we repeated our initial evaluation by involving only those participants who had serum creatinine measures (both at the time of enrollment and during follow-up) performed at Quest Diagnostics.

\section{Results}

The mean age of participants was 48 years, $57 \%$ were African American, 15\% reported lifetime use of opiates, and $22 \%$ reported lifetime use of cocaine (table 1 ). Individuals who reported lifetime use of opiates and cocaine were more likely to be male, African American, living in poverty, lacking health insurance, and with lower mean education than individuals without a history of drug use ( $p<0.001$ for all variables). They were more likely to report tobacco use and problem drinking than those who did not use opiates and cocaine $(\mathrm{p}<0.001$ for both variables). They were also more likely to have a history of Hepatitis C and Hepatitis B ( $p<0.001$ for both variables), although there was no association between opiate and cocaine use and HIV. Individuals who used 
Table 2. Multiple logistic regression analyses for association between lifetime opiate/cocaine use and reduced eGFR, prevalent albuminuria, or rapid eGFR decline

\begin{tabular}{|c|c|c|c|}
\hline Models & $\begin{array}{l}\text { Reduced eGFR }(\mathrm{n}=2,286) \\
\mathrm{n} \text { event }=115, \text { OR }(95 \% \mathrm{CI})\end{array}$ & $\begin{array}{l}\text { Prevalent albuminuria } \\
(\mathrm{n}=1,652) \mathrm{n} \text { event }=190 \\
\text { OR }(95 \% \mathrm{CI})\end{array}$ & $\begin{array}{l}\text { Rapid kidney function } \\
\text { decline }(\mathrm{n}=1,665) \\
\text { n events }=230 \\
\text { OR }(95 \% \mathrm{CI})\end{array}$ \\
\hline \multicolumn{4}{|c|}{ Lifetime opiate users vs. non-users } \\
\hline Crude & $1.63(1.00-2.65)$ & $1.02(0.78-1.33)$ & $1.18(0.81-1.72)$ \\
\hline Adjusted* & $2.71(1.50-4.89)$ & $1.20(0.83-1.73)$ & $0.86(0.70-1.04)$ \\
\hline Adjusted* & $1.40(0.87-2.24)$ & $1.80(1.29-2.51)$ & $0.93(0.60-1.45)$ \\
\hline
\end{tabular}

* Adjusted for age, gender, race, poverty, insurance, alcohol use, tobacco use, hepatitis B virus, hepatitis C virus, diabetes mellitus, and hypertension.

Table 3. Multiple logistic regression analyses for association between current opiate/cocaine use and prevalent CKD, rapid eGFR decline, or albuminuria

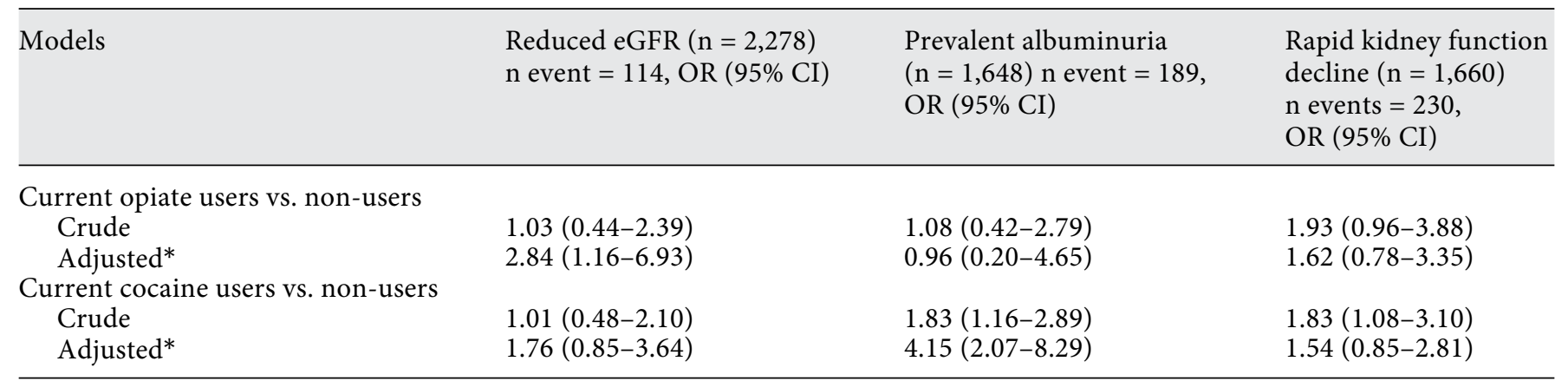

* Adjusted for age, gender, race, poverty, insurance, alcohol use, tobacco use, hepatitis B virus, hepatitis C virus, diabetes mellitus, and hypertension.

Among 2,278 participants available for the analyses of reduced eGFR, 78 reported current opiate use and 139 reported current cocaine use. Among the 1,648 participants available for the analyses of albuminuria, 49 reported current opiate use, and 75 reported current cocaine use. Among the 1,660 participants available for the analyses of rapid eGFR decline, 56 reported current opiate use, and 100 reported current cocaine use.

cocaine were less likely to have diabetes $(p=0.001)$, but there was no association between diabetes and opiate use; those who used opiates were less likely to have hypertension $(\mathrm{p}=0.04)$, but there was no association between hypertension and cocaine use.

A total of 115 (5\%) participants had reduced eGFR, 190 (11.5\%) had albuminuria, and 230 (13.8\%) had rapid kidney function decline (table 2). After adjusting for age, gender, race, poverty, insurance, smoking status, problem drinking, hypertension, diabetes, HIV status, Hepatitis C and Hepatitis B infection, participants with a history of opiate use had a greater odds of reduced eGFR than those without a history of drug use (OR 2.71, 95\% CI 1.50-4.89). A similar pattern was seen for lifetime cocaine use and re- duced eGFR, but this was not statistically significant (OR $1.40,95 \%$ CI $0.87-2.24$ ). Participants with a history of cocaine use had a greater adjusted odds of albuminuria (OR $1.80,95 \%$ CI 1.29-2.51), but the relationship between opiate use and albuminuria was not statistically significant (OR 1.20, 95\% CI 0.83-1.73). Lifetime opiate and cocaine use was not associated with increased adjusted odds of rapid kidney function decline (OR 0.86, 95\% CI 0.70-1.04 for opiates; OR $0.93,95 \%$ CI $0.60-1.45$ for cocaine).

\section{Sensitivity Analyses}

Current drug use data were available for 2,278, 1,648, and 1,660 participants for reduced eGFR, albuminuria, and rapid eGFR decline outcomes, respectively (table 3 ). 
After adjustment, individuals currently using opiates were more likely to have reduced eGFR than those not currently using them (OR 2.84, 95\% CI 1.16-6.93). A similar pattern, although not statistically significant, was seen for those currently using cocaine (OR 1.76, 95\% CI 0.853.64). Those currently using cocaine were more likely to have increased adjusted odds of albuminuria (OR 4.15, 95\% CI 2.07-8.29), but there was no statistically significant relationship between current opiate use and albuminuria. Similar to our primary analysis, current opiate and cocaine use was not associated with adjusted odds of rapid kidney function decline. Analyses restricted to participants with all labs drawn at Quest Diagnostics yielded results that were similar to those of the primary analyses (data not shown).

\section{Discussion}

Among urban-dwelling adults, we found that there was an association between opiate and cocaine use and adverse kidney outcomes. Specifically, lifetime opiate use was independently associated with prevalent reduced kidney function, and lifetime cocaine use was associated with albuminuria. Lifetime cocaine use had a similar relationship with reduced kidney function, as did historical opiate use and albuminuria, although these findings were not statistically significant. Lifetime opiate and cocaine use was not associated with rapid kidney function decline over 5 years of follow-up. Examination of current opiate and cocaine use revealed similar findings to those found with lifetime use, but with greater magnitude of the associations. Thus, lifetime or current use of opiates and cocaine may be risk factors for reduced kidney function and/or albuminuria.

To our knowledge, this is the first population-based prospective study to examine the relationship between opiate and cocaine use and kidney outcomes in an urban population, and to extend the findings of prior investigations. A prospective study by Vupputuri et al. [8] among hypertensive men at the Veterans Administration found that ongoing use of cocaine and psychedelic drugs was associated with 3-3.9 times increased odds of developing mild kidney function decline (defined as a rise in creatinine of 0.6 or greater). In a case-control study comparing adults with ESRD to controls in the general population, Perneger et al. [14] found that those with a history of opiate use were 19.1 times more likely to develop ESRD than those without prior use. Norris et al. [15] compared the history of cocaine use in hemodialysis patients with and

Opiate and Cocaine Use and CKD in

Urban Adults without hypertension-associated ESRD (HTN-ESRD), and found that those who used cocaine were 9.44 times more likely to have HTN-ESRD than those who never used. However, Akkina et al. [7] examined data from the National Health and Nutrition Examination Survey (NHANES), and found no difference in prevalence of CKD (defined as reduced eGFR and/or albuminuria) among those with or without a history of lifetime drug use. These studies, and ours, suggest that the strength of the association of drug use with kidney disease outcomes may vary by the temporal relation of drug use with the assessment of outcomes, and may vary across demographic and clinical populations. For example, the differences seen between our findings and those of Akkina et al. [7] may reflect variability in the timing of drug use, study populations and setting. Akkina et al. [7] evaluated the relationship between reported lifetime use but did not specifically look at current or ongoing drug use. NHANES included both urban- and non-urban-dwelling adults of whom $76 \%$ were white, whereas HANDLS participants were all urban dwelling and 57\% self-identified as African American. The type of drugs examined also differed among the study populations; Akkina et al. [7] included only heroin use in their analysis of opiates, whereas we examined heroin, morphine, and codeine. Thus, our conflicting findings regarding opiates may be partially influenced by the misuse of prescription narcotics among HANDLS participants.

The pathophysiologic link between drug use and kidney disease is poorly defined. Cocaine inhibits the presynaptic reuptake of catecholamines, thereby increasing the stimulation of sympathetically innervated tissues and adrenal norepinephrine and epinephrine secretion [16-18]. Potential renal injury occurs due to vasoconstriction and alteration of glomerular microcirculation via the actions of norepinephrine, endothelins $[16,17,19]$, the renin-angiotensin-aldosterone system $[16,20]$, and the L-arginine-nitric oxide pathway $[16,21]$. Cocaine has also been shown to increase oxidative stress and reduce intracellular glutathione in renal epithelial cells [22]. However, pathologic lesions described in rat studies [23] and human autopsy reports [24] vary greatly. Thus, the exact mechanism of injury from cocaine and resulting pathology remains unclear.

Similarly, the pathophysiologic link between opiate use and kidney disease is incompletely understood. In vitro studies demonstrate that heroin's active metabolite, morphine, has direct effects on mesangial cell proliferation and matrix deposition, fibroblast proliferation, and macrophage activity [25-30]. Morphine triggers glomer- 
ular epithelial cell growth and apoptosis at lower and higher concentrations, respectively [31], and increases oxidative stress [26, 31-33]. In vivo data are lacking [16]. Historically, several pathologic lesions including focal segmental glomerulosclerosis, sclerosing glomerulonephritis, focal membranoproliferative glomerulonephritis, among others have been described in people who used opiates [16]. However, it has been difficult to separate the isolated impact of opiates from commonly co-occurring infections such as infective endocarditis, HIV, and hepatitis, all of which are associated with distinct renal pathologies. Renal injury might also arise from various drug adulterants and carriers, such as with levamisole in cocaine and associated vasculitis [34]. Amyloidosis has been documented in those using IV drugs in the setting of chronic skin infections and inflammation due to 'skin popping' [16, 35-37]. Thus, the exact relationship between opiate and cocaine use and kidney disease is likely multifactorial, resulting from the substances, adulterants or drug carriers, and co-occurring hemodynamic, inflammatory and infectious processes.

Our study had limitations. First, we lacked complete data on albuminuria and follow-up eGFR, and relied on self-report of drug use, which may have been subject to underreporting. We also relied on a single measure of albuminuria, which may have misclassified persons with only transient albuminuria. Second, our focus on lifetime use, defined as at least 5 times over the life span, may not have captured the potential individual impact of cumulative amount used or pattern of use over time. Our sensitivity analyses of current use, however, did support the primary findings of our study. Third, we examined a relatively short duration of follow-up (5 years), which may not have fully captured the impact of opiate and cocaine use on persons with preserved kidney function at baseline. Fourth, the results from our study may not be generalizable to non-urban populations. Our study focused on morphine derivatives, and thus does not capture the potential impact of several commonly used opioids including oxycodone, methadone and fentanyl. Fifth, it is unclear if our findings reflect a causative link between opiate use and reduced eGFR, or rather the increased use of opiates in this population due to limited alternative options for pain control because of recommended avoidance of non-steroidal anti-inflammatory agents in CKD patients. Sixth, selected participants were less likely to report lifetime use of cocaine and opiates than participants in the overall HANDLS study; thus, we likely provided a conservative estimate of opiate and cocaine use in Baltimore, which may have influenced the observed as- sociations with kidney outcomes. Finally, while we adjusted for several potential confounders of the association of opiate and cocaine use and kidney outcomes, residual confounding is likely.

The limitations of our study are balanced by its prospective design and our focus on a high-risk, urban population. The challenge henceforth lies in how to address this potentially modifiable risk factor for kidney disease. Both opiate use and cocaine use were associated with living below the federal poverty level and lack of health insurance at study baseline. Thus, people who use opiates and cocaine may encounter barriers to care- and health-promoting behaviors that amplify the detriment they face as a direct consequence of substance use. Further and continued support for understanding community-based substance use disorders is needed, as are public policies that prioritize the availability of these services. Additional research is warranted investigating the impact of opiate and cocaine use on kidney outcomes, and on the interaction between drug use and other known risk factors for CKD.

\section{Acknowledgments}

The authors would like to thank the participants of the HANDLS study.

\section{Support and Financial Disclosure}

This work was supported by the Intramural Research Program of the National Institute on Aging, National Institutes of Health (NIH). Dr. Deidra C. Crews was supported by grant K23DK097184 from the National Institute of Diabetes and Digestive and Kidney Diseases, NIH.

\section{References}

1 Center for Behavioral Health Statistics and Quality: 2014 National Survey on Drug Use and Health: Detailed Tables. Rockville, Substance Abuse and Mental Health Services Administration, 2015.

2 Substance Abuse and Mental Health Services Administration. Behavioral Health Barometer: Maryland, 2014. HHS Publication No. SMA-15-4895MD. Rockville, Substance Abuse and Mental Health Services Administration, 2015.

3 Maryland Department of Health and Mental Hygiene. Drug and Alcohol Related Intoxication Deaths in Maryland, 2014. http://bha. dhmh.maryland.gov/OVERDOSE_PREVENTION/Documents/2015.05.19\%20-\%20 Annual\%20OD\%20Report \%202014 merged\%20file\%20final.pdf. 
4 Behavioral Health System Baltimore. Baltimore Mayor's Heroin Treatment \& Prevention Task Force, 2015. http://health.baltimorecity.gov/sites/default/files/Mayor\%20Heroin $\% 20$ Treatment $\% 20$ Prevention $\% 20$ Task\%20Force\%20Final\%20Report\%20 July\%2013\%202015.pdf.

5 National Center for Statistics and Analysis: 2013 Motor Vehicle Crashes: Overview. (Traffic Safety Facts Research Note. Report No. DOT HS 812 101). Washington, National Highway Traffic Safety Administration, 2014.

6 US Renal Data System: USRDS 2012 Annual Data Report: Atlas of Chronic Kidney Disease and End-Stage Renal Disease in the United States. Bethesda, National Institutes of Health, National Institute of Diabetes and Digestive and Kidney Diseases, 2012.

7 Akkina SK, Ricardo AC, Patel A, Das A, Bazzano LA, Brecklin C, et al: Illicit drug use, hypertension, and chronic kidney disease in the US adult population. Transl Res 2012;160: 391-398.

8 Vupputuri S, Batuman V, Muntner P, Bazzano LA, Lefante JJ, Whelton PK, et al: The risk for mild kidney function decline associated with illicit drug use among hypertensive men. Am J Kidney Dis 2004;43:629-635.

9 Evans MK, Lepkowski JM, Powe NR, LaVeist T, Kuczmarski MF, Zonderman AB: Healthy aging in neighborhoods of diversity across the life span (HANDLS): overcoming barriers to implementing a longitudinal, epidemiologic, urban study of health, race, and socioeconomic status. Ethn Dis 2010;20:267-275.

10 Crews DC, Kuczmarski MF, Grubbs V, Hedgeman E, Shahinian VB, Evans MK, et al: Effect of food insecurity on chronic kidney disease in lower-income Americans. Am J Nephrol 2014;39:27-35.

11 Crews DC, Kuczmarski MF, Miller ER 3rd, Zonderman AB, Evans MK, Powe NR: Dietary habits, poverty, and chronic kidney disease in an urban population. J Ren Nutr 2015; 25:103-110.

12 Levey AS, Stevens LA, Schmid CH, Zhang YL, Castro AF 3rd, Feldman HI, et al: A new equation to estimate glomerular filtration rate. Ann Intern Med 2009;150:604-612.

13 Hiramoto JS, Katz R, Peralta CA, Ix JH, Fried $\mathrm{L}$, Cushman M, et al: Inflammation and coagulation markers and kidney function decline: the multi-ethnic study of atherosclero- sis (MESA). Am J Kidney Dis 2012;60:225232.

14 Perneger TV, Klag MJ, Whelton PK: Recreational drug use: a neglected risk factor for end-stage renal disease. Am J Kidney Dis 2001;38:49-56.

15 Norris KC, Thornhill-Joynes M, Robinson C, Strickland T, Alperson BL, Witana SC, et al: Cocaine use, hypertension, and end-stage renal disease. Am J Kidney Dis 2001;38:523528.

16 Jaffe JA, Kimmel PL: Chronic nephropathies of cocaine and heroin abuse: a critical review. Clin J Am Soc Nephrol 2006;1:655-667.

17 Nzerue CM, Hewan-Lowe K, Riley LJ Jr: Cocaine and the kidney: a synthesis of pathophysiologic and clinical perspectives. Am J Kidney Dis 2000;35:783-795.

18 Chiueh CC, Kopin IJ: Centrally mediated release by cocaine of endogenous epinephrine and norepinephrine from the sympathoadrenal medullary system of unanesthetized rats. J Pharmacol Exp Ther 1978;205:148154.

19 Kon V, Yoshioka T, Fogo A, Ichikawa I: Glomerular actions of endothelin in vivo. J Clin Invest 1989;83:1762-1767.

20 Hendricks-Munoz KD, Gerrets RP, Higgins RD, Munoz JL, Caines VV: Cocaine-stimulated endothelin-1 release is decreased by angiotensin-converting enzyme inhibitors in cultured endothelial cells. Cardiovasc Res 1996; 31:117-123.

21 Mo W, Singh AK, Arruda JA, Dunea G: Role of nitric oxide in cocaine-induced acute hypertension. Am J Hypertens 1998;11(6 pt 1):708-714.

22 Palamara AT, Di Francesco P, Ciriolo MR Buè $\mathrm{C}$, Lafavia E, Rotilio $\mathrm{G}$, et al: Cocaine increases Sendai virus replication in cultured epithelial cells: critical role of the intracellular redox status. Biochem Biophys Res Commun 1996;228:579-585.

23 Barroso-Moguel R, Mendez-Armenta $M$, Villeda-Hernandez J: Experimental nephropathy by chronic administration of cocaine in rats. Toxicology 1995;98:41-46.

24 Di Paolo N, Fineschi V, Di Paolo M, Wetly CV, Garosi G, Del Vecchio MT, et al: Kidney vascular damage and cocaine. Clin Nephrol 1997;47:298-303.

25 Singhal PC, Gibbons N, Abramovici M: Long term effects of morphine on mesangial cell proliferation and matrix synthesis. Kidney Int 1992;41:1560-1570.

26 Singhal PC, Pamarthi M, Shah R, Chandra D, Gibbons N: Morphine stimulates superoxide formation by glomerular mesangial cells. Inflammation 1994; 18:293-299.

27 Singhal PC, Pan CQ, Sagar S, Gibbons N, Valderrama E: Morphine enhances deposition of ferritin-antiferritin complexes in the glomerular mesangium. Nephron 1995;70: 229-234.

28 Singhal PC, Mattana J, Garg P, Arya M, Shan Z, Gibbons N, et al: Morphine-induced macrophage activity modulates mesangial cell proliferation and matrix synthesis. Kidney Int 1996;49:94-102.

29 Singhal PC, Sharma P, Kapasi AA, Reddy K, Franki N, Gibbons N: Morphine enhances macrophage apoptosis. J Immunol 1998;160: 1886-1893.

30 Singhal PC, Sharma P, Sanwal V, Prasad A, Kapasi A, Ranjan R, et al: Morphine modulates proliferation of kidney fibroblasts. Kidney Int 1998;53:350-357.

31 Patel K, Bhaskaran M, Dani D, Reddy K, Singhal PC: Role of heme oxygenase-1 in morphine-modulated apoptosis and migration of macrophages. J Infect Dis 2003; 187: 47-54.

32 Sharp BM, Keane WF, Suh HJ, Gekker G, Tsukayama D, Peterson PK: Opioid peptides rapidly stimulate superoxide production by human polymorphonuclear leukocytes and macrophages. Endocrinology 1985;117:793-795.

33 Maines MD: Heme oxygenase: function, multiplicity, regulatory mechanisms, and clinical applications. FASEB J 1988;2:2557-2568.

34 Garg L, Gupta S, Swami A, Zhang P: Levamisole/cocaine induced systemic vasculitis and immune complex glomerulonephritis. Case Rep Nephrol 2015;2015:372413

35 Neugarten J, Gallo GR, Buxbaum J, Katz LA, Rubenstein J, Baldwin DS: Amyloidosis in subcutaneous heroin abusers ('skin poppers' amyloidosis'). Am J Med 1986;81:635-640.

36 Crowley S, Feinfeld DA, Janis R: Resolution of nephrotic syndrome and lack of progression of heroin-associated renal amyloidosis. Am J Kidney Dis 1989;13:333-335.

37 Dubrow A, Mittman N, Ghali V, Flamenbaum W: The changing spectrum of heroinassociated nephropathy. Am J Kidney Dis 1985;5:36-41. 